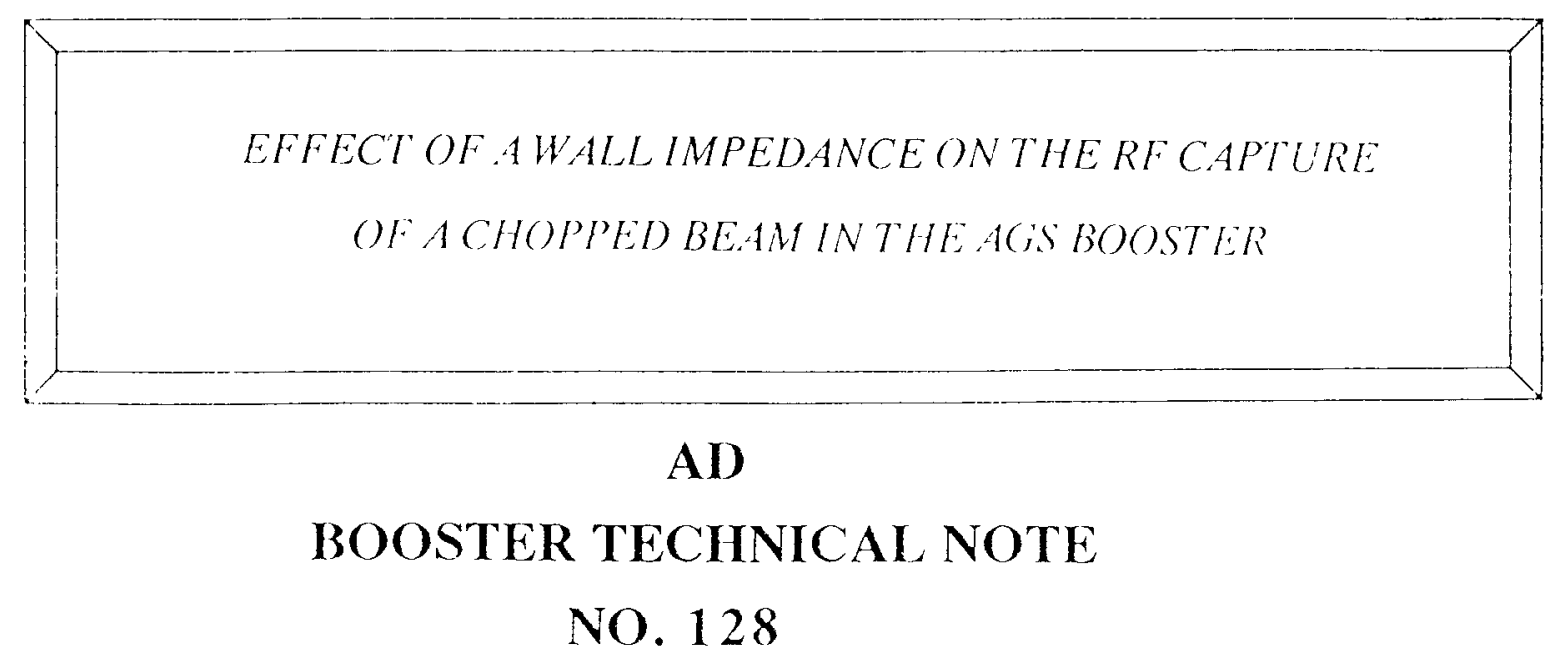

F. KHIARI, A. LUCCIO AUGUST 22, 1988

ACCELERTOR DEVEL OPMENT DEPARTMENT BROOKIIAVEN NATIONAL IABORATORY

UPTON. NEW YORK 11973 


\title{
EFFECT OF A WALL IMPEDANCE ON THE RF CAPTURE OF A CHOPPED BEAM IN THE AGS BOOSTER
}

\author{
F.Z. Khiari and A.U. Luccio \\ Accelerator Development Department, \\ Brookhaven National Laboratory, \\ Upton, New York 11973
}

\section{Introduction}

The AGS Booster is designed to rapidly accelerate high intensity beams $\left(1.510^{13}\right)$ of protons to a kinetic energy of $1.5 \mathrm{GeV}$ and inject them into the AGS. Due to the high beam currents present in the machine, even a small beam loss during the rf capture will result in unacceptably high background radiation around the accelerator ring. To avoid this problem, it is necessary to capture the maximum number of injected particles. In a previous note ${ }^{[1]}$, we presented the results of a series of simulation studies of proton $\mathrm{rf}$ capture in the AGS Booster in the presence of space charge. We concluded from those studies that it was necessary to chop the incoming beam from the Linac in order to achieve a capture efficiency greater than $90 \%$. To this effect, the computer code ESME ${ }^{[2]}$ was further improved to simulate the capture process of a chopped beam. In this note, we report on a new series of simulation studies of proton if capture in the AGS Booster for the case of a chopped beam in the presence of a wall impedance.

\section{RF Capture of a Chopped Beam with Space Charge ${ }^{[3]}$}

We studied 6 cases which we will label 1, 2, 3, 4A, 4B, and 4C. All the cases were done for the voltage program shown in Fig. 1 and for an initial random uniform azimuthal 
distribution and a gaussian energy distribution with $\sigma_{E} \approx 0.2 \mathrm{MeV}$ at an injection energy of $200 \mathrm{MeV}$ for cases $1-4 \mathrm{~B}$ and $201 \mathrm{MeV}$ for case $4 \mathrm{C}$. We found the voltage curve in Fig. 1 to be near optimum for both early capture and later acceleration. The magnetic field used in the different cases was given by

$$
B(t)=B_{i}+\left(B_{f}-B_{i}\right)\left(\frac{t-t_{i}}{t_{f}-t_{i}}\right)^{\alpha},
$$

where $B_{i}=0.16 T$ and $B_{f}=0.54 T$ are the initial and final magnetic fields defined at times $t_{i}=0$ and $t_{f}=60 \mathrm{~ms}$ respectively. The coefficient $\alpha$ was equal to 2 for case $1,3 / 2$ for case $2,5 / 4$ for case 3 , and 1 for cases $4 \mathrm{~A}-4 \mathrm{C}$. The curves of the magnetic field programs are shown in Fig. 2.

One first simulation was made for the case where we chopped $5^{\circ}$ on each side of every bunch of the injected Linac beam (cases $1-4 \mathrm{~A}$ ). This amounts to $\approx 8 \%$ of the total beam delivered by the source. The bucket areas and rf captures for theses cases are listed in Table 1. Notice that the rf captures for cases 1,2 and 3 are all high but the rf capture for case $4 \mathrm{~A}$ is unacceptably small. This is due to the fact that $\dot{B}(t)$, given by

$$
\dot{B}(t)=\alpha\left(B_{f}-B_{i}\right) \frac{t^{\alpha-1}}{t_{f}^{\alpha}}
$$

starts from zero and increases more or less slowly therefrom for cases 1, 2 and 3 whereas it has a constant value of $\approx 6.4 \mathrm{~T} / \mathrm{s}$ during the whole cycle for case $4 \mathrm{~A}$. This makes the bucket area and the rf capture at injection for case $4 \mathrm{~A}$ much smaller than those of cases 1,2 and 3. To illustrate this, we show the particle distribution in phase-space and the rf bucket at the beginning of injection for cases $1-3$ and $4 \mathrm{~A}$ in Fig. 3a and Fig. $3 \mathrm{~b}$ respectively. This demonstrates the fact that during capture, the rate of rise of the magnetic field should be kept as low as possible to be able to create the largest bucket area for a given initial voltage. With large $\dot{B}$, as for case $4 \mathrm{~A}$, chopping the beam loses its advantage since the bucket area is reduced due to the large value of $\dot{B}$. 
To improve the rf capture for case $4 \mathrm{~A}$, we chopped more beam. We studied case $4 \mathrm{~B}$, shown in Fig. 4, where we chopped $\approx 30^{\circ}$ on one side of each bunch of the injected beam and $\approx 17^{\circ}$ on the other. This corresponds to $\approx 50 \%$ of the total beam from the source. This, of course, has improved the rf capture $(\approx 80 \%)$, but not enough to bring it to the level of cases $1-3$. The difference in this case can be accounted for by the bucket motion during the $100 \mu \sec (\approx 84$ turns) injection time. This motion is related to the energy gain per turn of the synchronous particle which is given by

$$
\Delta E / \operatorname{Turn}[M e V]=2 \pi \rho R \dot{B} 10^{-6}
$$

where $\rho=13.75 \mathrm{~m}$ is the curvature radius of the Booster dipole magnets and $\ddot{R}=$ $32.114 m$ is the average radius of the equilibrium orbit. With $\dot{B} \approx 6.4 T / s$, we get $\triangle E / T u r n \approx 17.7 \mathrm{kV}$. Therefore, the bucket moves up in energy by about $1.5 \mathrm{MeV}$ during the 84 turns it takes to finish injection.

Case $4 \mathrm{C}$ shows the effect of injection of the chopped Linac beam at $201 \mathrm{MeV}$ kinetic energy instead of $200 \mathrm{MeV}$. We show in Fig. 5 the evolution of the beam-rf bucket system during the first $100 \mu s e c$ of the multiturn injection for case $4 \mathrm{C}$. This finally has made the rf capture at $500 \mu \mathrm{sec}$ and $1 \mathrm{~ms}$ very close to that of cases $1-3$ (see Table 1). However, the overall capture efficiency from the Linac drops from $92 \%$ to $50 \%$, a price too high to be affordable.

\section{Wall Impedance Effects}

In the capture studies presented so far, we included the rf focusing and the space charge forces only. Some studies have also been made to evaluate the contribution from the wall impedances. This wall impedance $\left(Z^{W}(\omega)\right)$ combines with the space charge impedance $\left(Z^{s c}(\omega)\right)$ to induce a voltage

$$
V^{i}(\theta)=\sum_{n} Z\left(n \omega_{r f}\right) I_{n}(\theta)
$$

where $Z \equiv Z^{s c}+Z^{I V}$ and $I_{n}(\theta)$ is the $n^{t h}$ complex Fourier component of the beam current 


$$
I_{n}(\theta)=e N \omega_{r f} a_{n} e^{i\left(n \theta+\theta_{n}\right)} .
$$

$\mathrm{N}$ is the number of particles per bunch and $a_{n}$ and $\theta_{n}$ are the real amplitude and phase of the $n^{t h}$ component of the spectrum of the beam current. The resulting energy change is then

$$
\delta E(\theta)=\Re\left(e V^{i}(\theta)\right)=e^{2} N \omega_{r f} \Re \sum_{n}\left[Z^{W}\left(n \omega_{r f}\right)+i \frac{n Z_{\circ} g}{2 \beta \gamma^{2}}\right] a_{n} e^{i\left(n \theta+\theta_{n}\right)},
$$

where $\Re$ denotes the real part and we use the convention $i=-j$. Expressing $Z^{W}$ as $\Re Z^{W}+i \Im Z^{W}$ we get

$$
\delta E(\theta)=e^{2} N \omega_{r f} \Re \sum_{n}\left[\Re Z^{W}\left(n \omega_{r f}\right)+i\left[\Im Z^{W}\left(n \omega_{r f}\right)+\frac{n Z_{\circ} g}{2 \beta \gamma^{2}}\right]\right] a_{n} e^{i\left(n \theta+\theta_{n}\right)}
$$

$Z^{W}$ can also be expressed as

$$
Z^{W}=\left|Z^{W}\right| e^{i \chi}
$$

where $\chi$ is the wall impedance phase angle. The real and imaginary parts of $Z^{W}$ are then given by

$$
\Re Z^{W}=\left|Z^{W}\right| \cos \chi
$$

and

$$
\Im Z^{W}=\left|Z^{W}\right| \sin \chi
$$

The energy change can then be expressed as

$$
\delta E(\theta)=e^{2} N \omega_{r f} \sum_{n} a_{n}\left|Z\left(n \omega_{r f}\right)\right| \cos \left(n \theta+\theta_{n}+\varphi_{n}\right)
$$


where

$$
\left|Z\left(n \omega_{r f}\right)\right|=\sqrt{\left|Z^{W}\left(n \omega_{r f}\right)\right|^{2} \cos ^{2} \chi+\left[\left|Z^{W}\left(n \omega_{r f}\right)\right| \sin \chi+\frac{n Z_{\circ} g}{2 \beta \gamma^{2}}\right]^{2}}
$$

and

$$
\varphi_{n}=\tan ^{-1} \frac{\left|Z^{W}\left(n \omega_{r f}\right)\right| \sin \chi+n Z_{\circ} g / 2 \beta \gamma^{2}}{\left|Z^{W}\left(n \omega_{r f}\right)\right| \cos \chi} .
$$

Elaborating on case $4 \mathrm{C}$, we studied the rf capture for 5 additional cases in which we varied $\left|Z^{W}\right|$ and $\chi$. We label these cases $5 \mathrm{~A}-\mathrm{B}$ and $6 \mathrm{~A}-\mathrm{C}$ and show in Fig. 7 a log-plot of the wall impedance used in the simulations. Since we bin the particles in 128 bins, we can only determine a maximum of 64 harmonics of the beam current. Therefore the beam harmonic number $n \equiv \omega / \omega_{r f}$ varies from 1 for the fundamental to 64 for the highest harmonic. Table 2 lists the various cases and their respective rf capture efficiencies at selected times during the early part of the cycle. Notice that the wall impedance for cases $5 \mathrm{~A}$ and $5 \mathrm{~B}\left(\left|Z^{W}\right|=200 \Omega, n=1-6 ;\left|Z^{W}\right|\right.$ is higher for higher beam harmonics $)$ does not affect the rf capture. The space charge contribution is still the dominant one. However, the wall impedance for cases $6 \mathrm{~A}-6 \mathrm{C}\left(\left|Z^{W}\right|=20 \mathrm{k} \Omega, n=1-6\right)$ does have an effect on the rf capture and this effect depends on the value of the angle $\chi$. Notice then that when $\chi=$ $\pi / 2$, the imaginary parts of the wall impedance and the space charge impedance add and their combined effect on the rf capture is strongest. There is $10 \%$ loss due to the wall impedance in this case. In contrast, when $\chi=-\pi / 2$, the imaginary parts of the space charge impedance and the wall impedance have opposite signs and their combined effect on the rf capture is weakest. In this case the maximum rf capture is comparable to the maximum if capture in the absence of the wall impedance. The rf capture for case $6 \mathrm{~B}$ is plotted in Fig. 8. The oscillations in the rf capture are attributed to bunch oscillations inside the of bucket as illustrated in Fig. 9. The mechanism behind these oscillations is not yet understood and needs further investigation. As expected, the rf capture for case 
$6 \mathrm{C}$ where $\chi=0$ lies somewherc between those of the previous two cases. In fact there is a $5 \%$ loss in this case.

To further illustrate the effect of the impedance phase angle $\chi$ on the if capture, we list in Table 3 the value of

$$
\sqrt{\left\langle(\delta E)^{2}\right\rangle}=\sqrt{\frac{1}{2 \pi} \int_{0}^{2 \pi}[\delta E(\theta)]^{2} d \theta}
$$

for cases $4 \mathrm{C}$ and $6 \mathrm{~A}-\mathrm{C}$. As expected, $\sqrt{\left\langle(\delta E)^{2}\right\rangle}$ is smallest for $\chi=-\pi / 2$. However, even though the peak induced voltage is highest for $\chi=\pi / 2$ (causing more beam loss), the oscillations in $\delta E$ from bin to bin are more numerous for $\chi=0$ thereby causing $\sqrt{<(\delta E)^{2}>}$ to be largest for this last case.

\section{Conclusions and discussions}

From the studies presented in this note, some conclusions can be drawn:

1. By chopping the Linac beam, we eliminate those particles that are close to the unstable fixed point of the rf bucket which results in a higher capture.

2. The case with a chopped beam and a slow magnetic field rise during capture (cases 1-3) gives a better capture efficiency because the bucket area is maximum for the voltage at injection.

3. The broad band wall impedance starts to affect the rf capture at $Z^{W W} \mid / n \geq 3 k \Omega$, but there is a noticcable dependence of the rf capture on the phase angle of the wall impedance with respect to the phase angle of the space charge impedance. When these two angles are opposite to each other, the bunches oscillate inside the rf buckets cansing the rf capture to oscillate. The highest losses $(10 \%)$ occur when the wall impedance and the space charge impedance have equal phase angles. The losses are reduced to $5 \%$ when these two impedances are at $90^{\circ}$ with respect each other. 


\section{REFERENCES}

[1] F. Z. Khiari, A. U. Luccio, W. T. Weng, ESME at BNL: Status Report and Simulation study of Proton rf Capture in the BNL Booster AD Booster Technical Note No. 118 (1988).

[2] J. A. MacLachlan, Particle Tracking in E- $\phi$ Space as a Design Tool for Cyclic Accelerators, Proceedings of The IEEE Particle Accelerator Conference, 1087 (1987).

3] F. Z. Khiari, A. U. Luccio, W. T. Weng, Simulation of Proton RF capture in the AGS Booster, BNL 41462. 


$\begin{array}{llll}\text { Case \# } & \alpha & 500 \mu \mathrm{sec} & 1 \mathrm{~ms} \\ 1 & 2 & 1.6 / 100 & - \\ 2 & 1.5 & 1.6 / 100 & 1.5 / 99 \\ 3 & 1.25 & 1.4 / 96 & 1.4 / 96 \\ 4 \mathrm{~A} & 1 & 1.1 / 55 & - \\ 4 \mathrm{~B} & 1 & 1.1 / 82 & 1.1 / 82 \\ 4 \mathrm{C} & 1 & 1.1 / 98 & 1.1 / 98\end{array}$

Table 1. List of Bucket Area[eV.sec]/Capture[\%] for cases 1-4C 


$\begin{array}{lllllll}\text { Case \# } & 4 \mathrm{C} & 5 \mathrm{~A} & 5 \mathrm{~B} & 6 \mathrm{~B} & 6 \mathrm{C} & 6 \mathrm{~A} \\ \left|Z^{W}\right|[k \Omega] & 0 & 0.2 & 0.2 & 20 & 20 & 20 \\ \chi[\mathrm{rad}] & - & -\pi / 2 & \pi / 2 & -\pi / 2 & 0 & \pi / 2 \\ & & & & & & \\ & & & \text { Capture }[\%] & & 59 \\ 0 \mu \text { sec } & 59 & 59 & 59 & 59 & 59 & 73 \\ 50 & 75 & 75 & 75 & 76 & 74 & 78 \\ 100 & 84 & 84 & 84 & 86 & 86 & 88 \\ 150 & 98 & 98 & 98 & 96 & 94 & 88 \\ 200 & 99 & 99 & 99 & 97 & 95 & 88 \\ 300 & 98 & 98 & 98 & 98 & 95 & 89 \\ 500 & 98 & 98 & 98 & 92 & 94 & 88 \\ 1 \text { msec } & 98 & 98 & 98 & 97 & 93 & \end{array}$

Table 2. List of Wall Impedances and RF Captures for Cases 4C, $5 \mathrm{~A}-\mathrm{B}$ and $6 \mathrm{~A}-\mathrm{C}$. 
Case \#

$100 \mu \mathrm{sec}$

500

1 msec
$4 C$

$6 \mathrm{~B}$

$6 \mathrm{C}$

$\sqrt{<(\delta E)^{2}>}[k e V]$

6

7

6
0.01

0.02

0.02
29

32

32
$6 \mathrm{~A}$

7

8

11

Table 3. List of $\sqrt{\left\langle(\delta E)^{2}>\right.}$ for cases $4 \mathrm{C}$ and $6 \mathrm{~A}-\mathrm{C}$. 


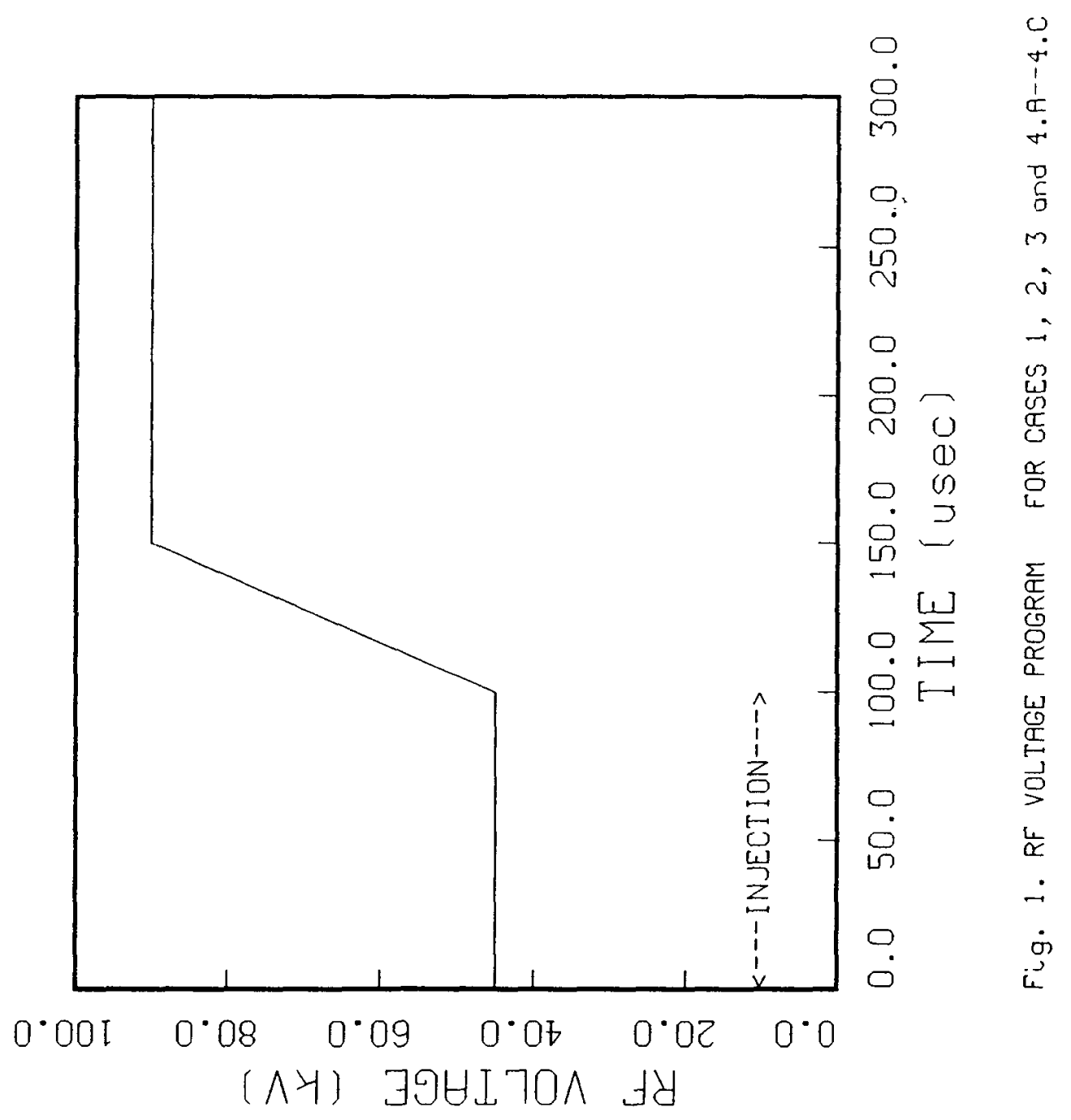




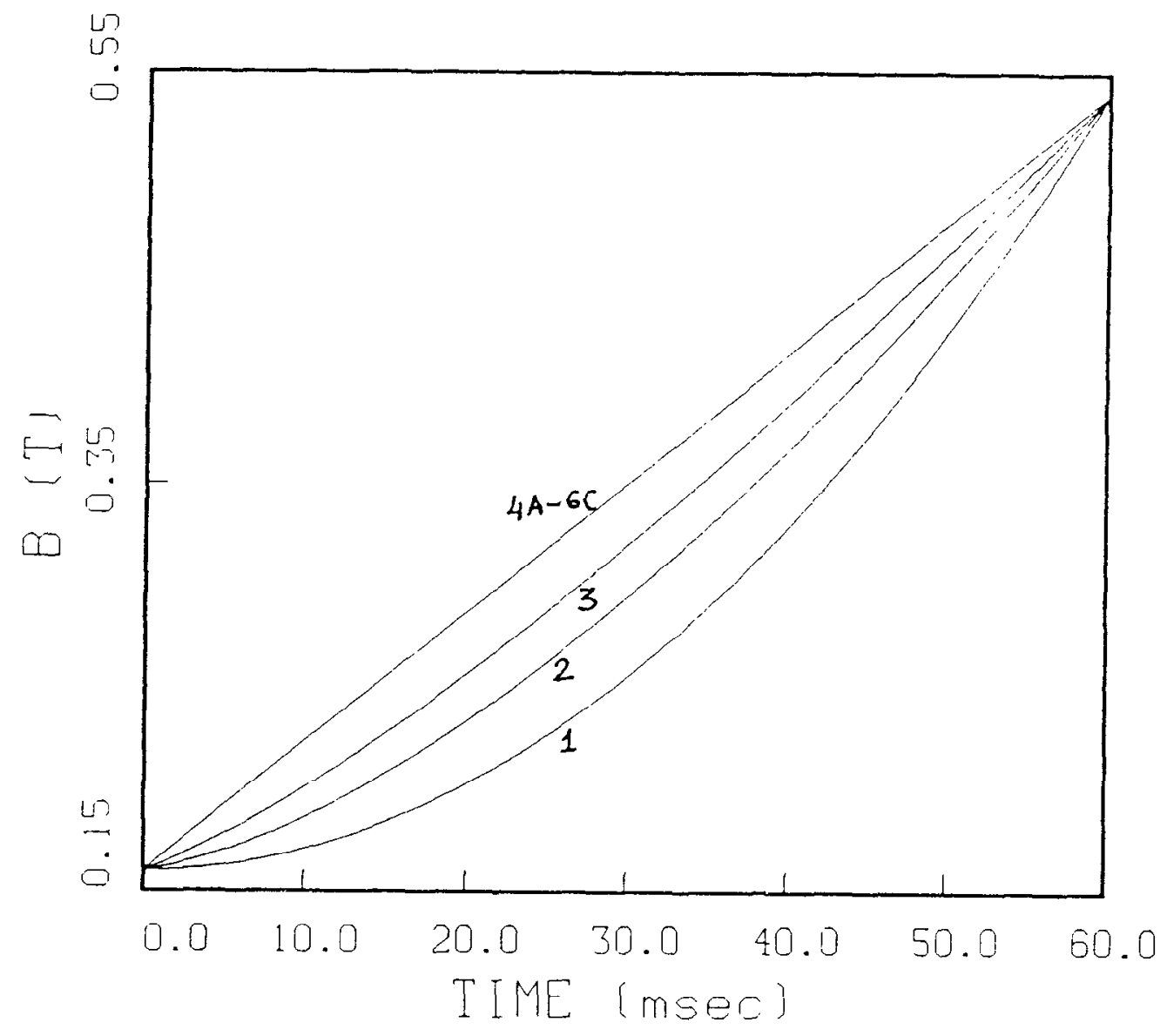

Fig. 2 B Curves for Cases $1-3$ and $4 \mathrm{~A}-6 \mathrm{C}$ 

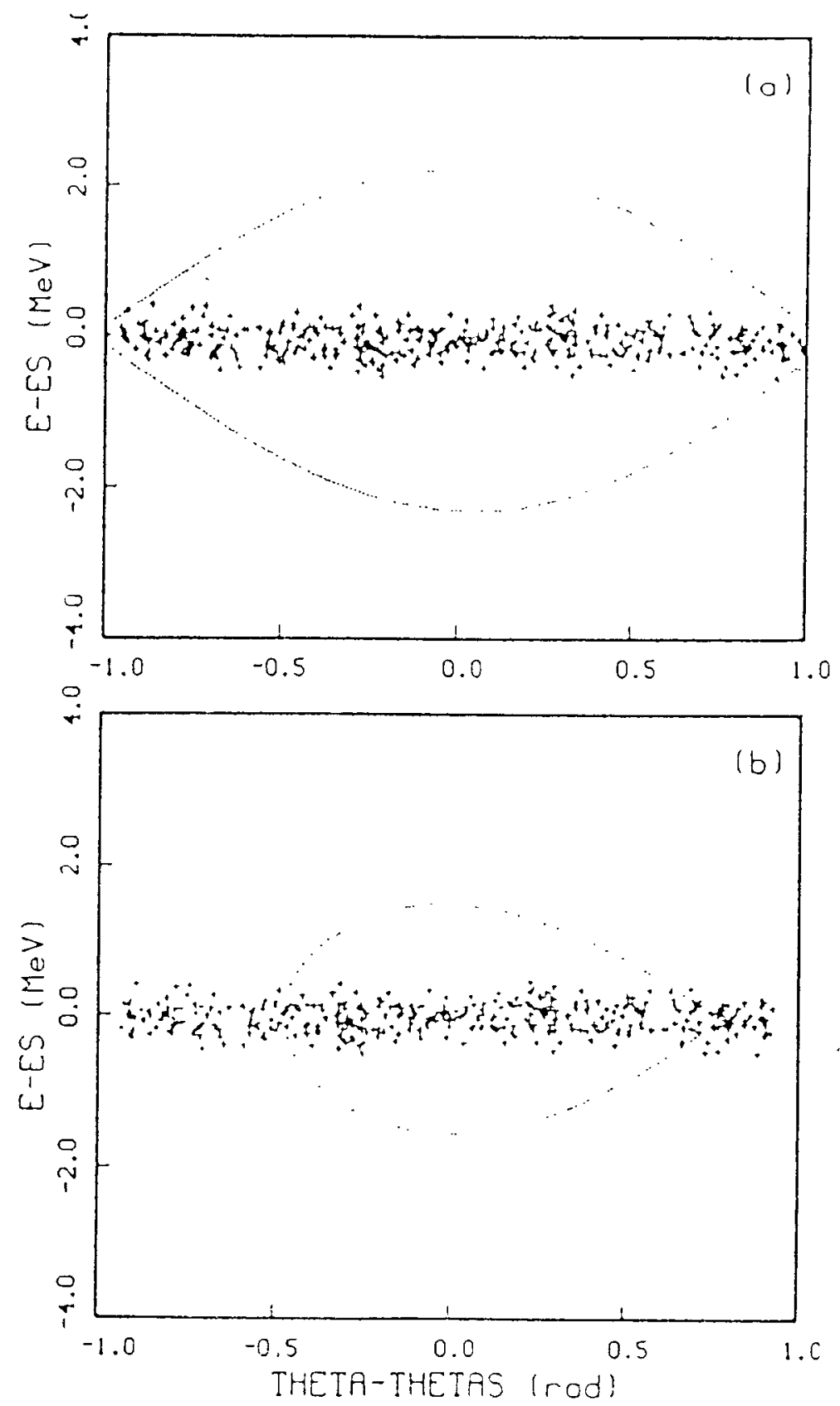

Fig. 3. a) Initial distribution and if bucket for cases 1-3. b) Case $4 . A$. 


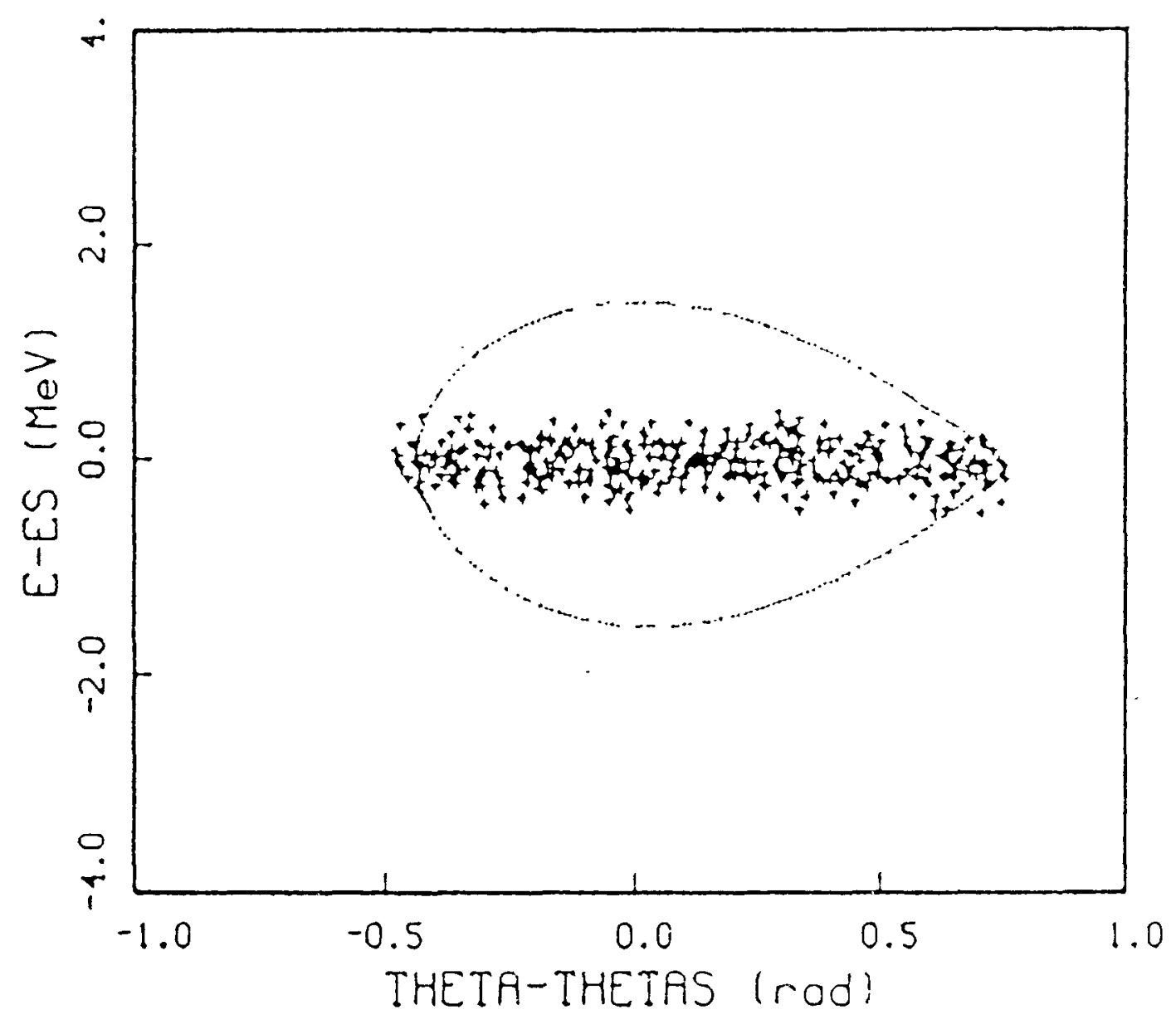

Fig. 4. Initial distribution and if bucket for case 4.B. 

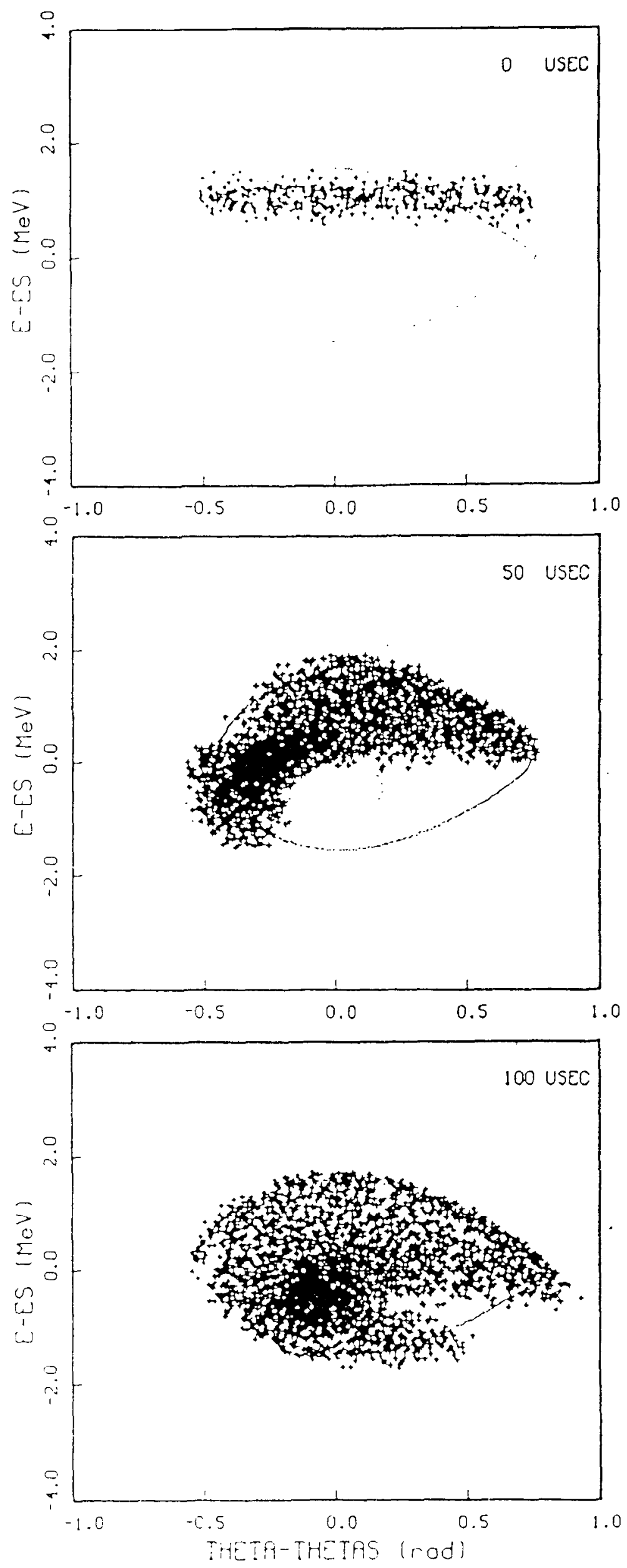

Fig. 5. Distribution for case 4.C at 0,50 , and $100 \mu \mathrm{sec}$. 


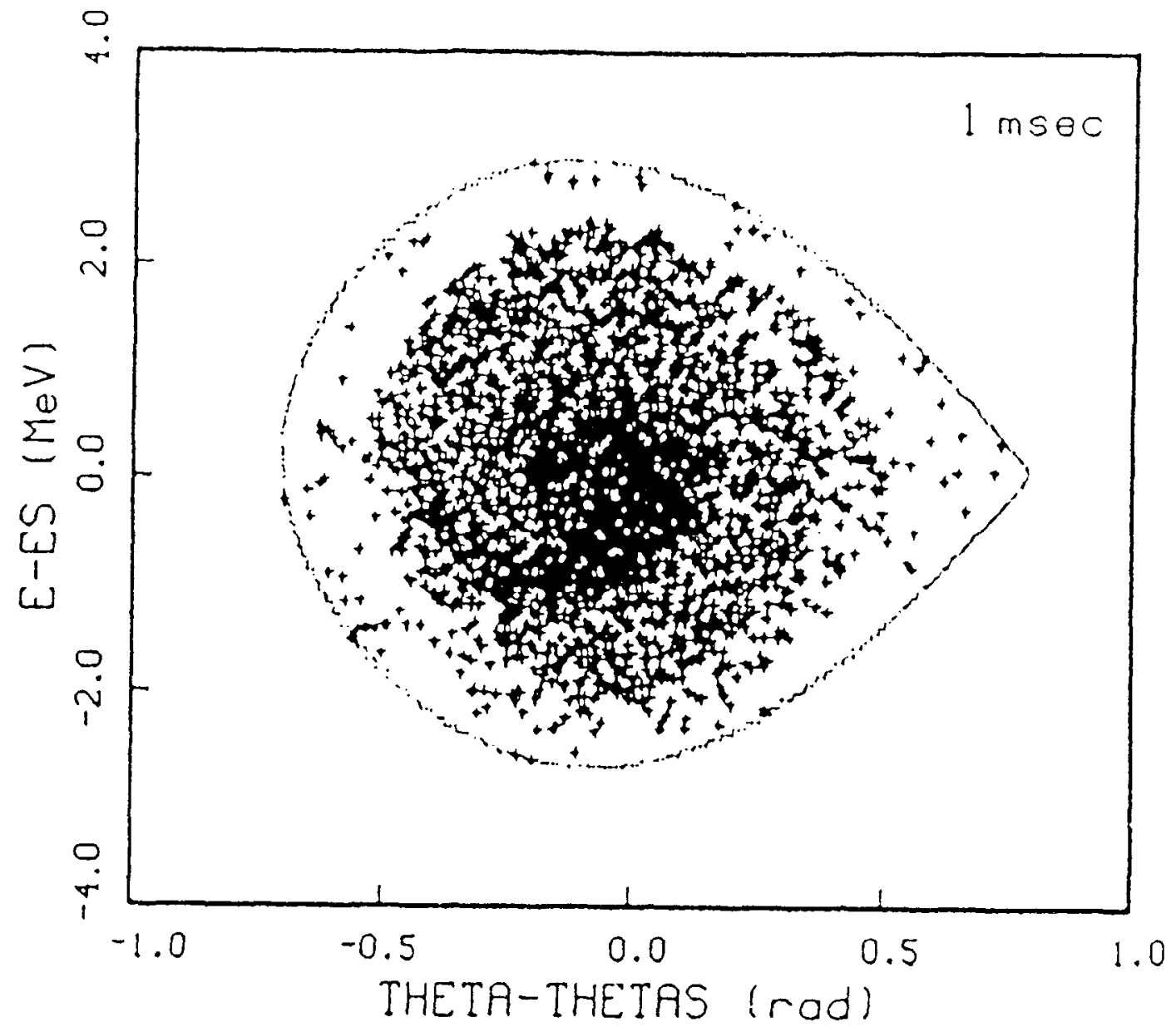

Fig. 6. Proton beam captured in the rf bucket at 1 msec. for case 4.C. 


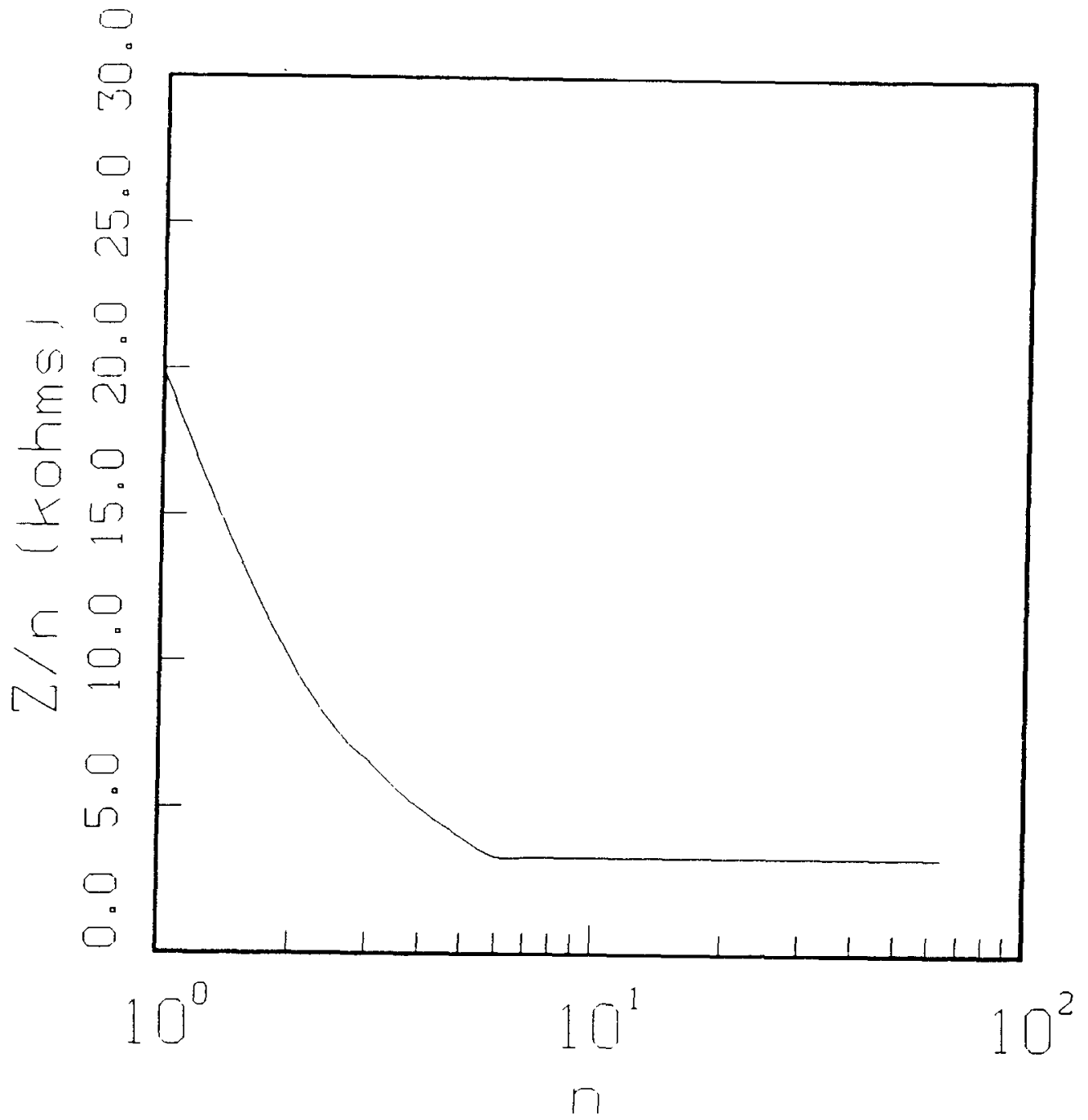

Fig. 7 Wall Impedance used in the Simulations of Cases $6 \mathrm{~A}$ 6C 


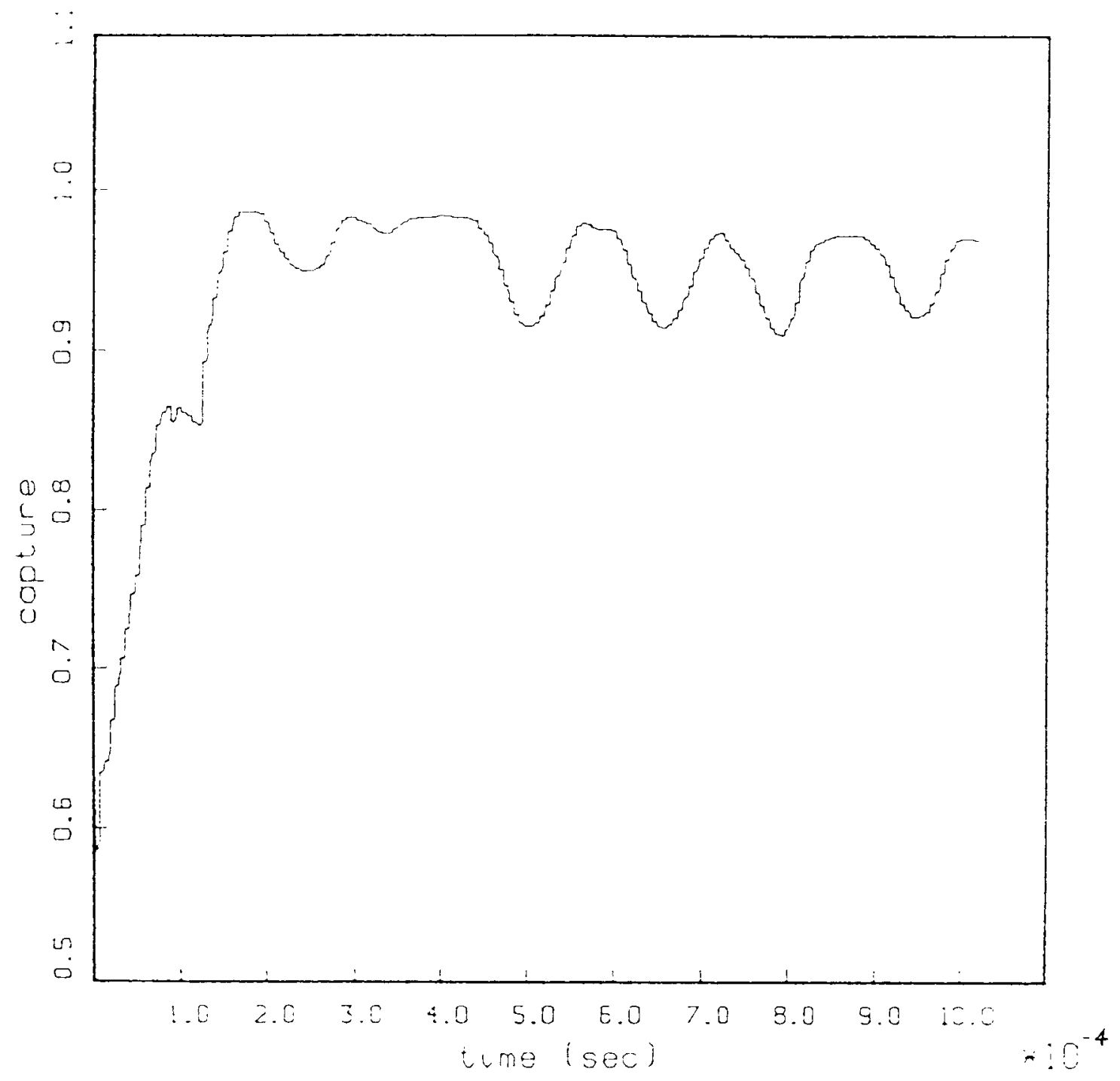

Fig. 8 Plot of the RF Capture for Case 6B $(\chi=0)$ 

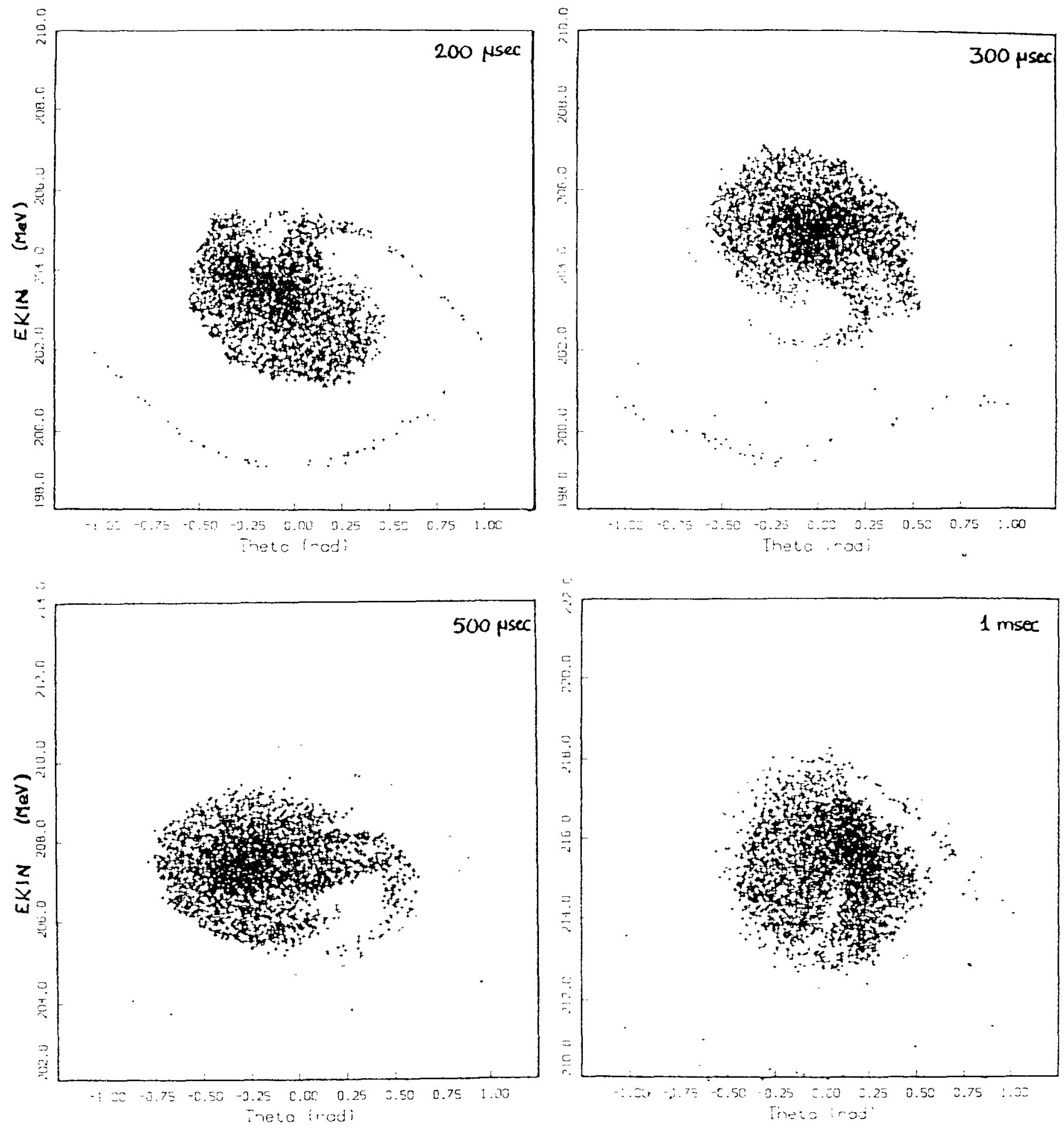

Fig. 9 Phase Space Plots for Case 6B showing the Bunch Oscillations inside the RF Bucket 